\title{
Fixed points of fuzzy neutrosophic soft mapping with decision-making
}

\author{
Muhammad Riaz and Masooma Raza Hashmi
}

${ }^{*}$ Correspondence:

mriaz.math@pu.edu.pk

Faculty of Science, Department of

Mathematics, University of the

Punjab, Lahore, Pakistan

\begin{abstract}
In this paper, we introduce some operations on a fuzzy neutrosophic soft set (fins-set) by utilizing the theories of fuzzy sets, soft sets and neutrosophic sets. We introduce $\mathfrak{f n} \mathfrak{s}$-mappings by using a cartesian product with relations on $\mathfrak{f n} \mathfrak{n}$-sets and establish some results on fixed points of an $\mathfrak{f n s}$-mapping. We present an algorithm to deal with uncertainties in the multi-criteria decision making to slenderize energy crises by using an $\mathfrak{f n} \mathfrak{s}$-average operator and a comparison table for $\mathfrak{f} \mathfrak{n} \mathfrak{s}$-sets.
\end{abstract}

MSC: 03B99; 03E99; 47H10

Keywords: $\mathfrak{f n} \mathfrak{s}$-set; $\mathfrak{f n} \mathfrak{n}$-cartesian product; $\mathfrak{f n} \mathfrak{n}$-mapping; fixed points of fnis-mapping; decision making

\section{Introduction}

Most of the problems in engineering, medical science, economics, environments etc. have various uncertainties. To deal with uncertainties there are different theories including the fuzzy set introduced by Zadeh [1], the soft set introduced by Molodtsov [2], the fuzzy soft

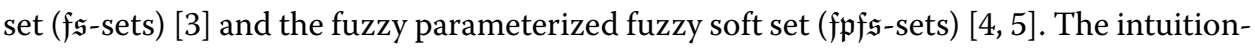
istic fuzzy set (if-set) introduced by Atanassov [6], which is an abstraction of fuzzy set. Smarandache [7] introduced the neutrosophic logic and neutrosophic set with its operations and some significant outcomes.

A fuzzy set [1] is a significant mathematical model to characterize an assembling of objects whose boundary is obscure. A soft set [2] is a mathematical tool to handle the uncertainties associated with real world data-based problems. Fuzzy parameterized fuzzy soft sets introduced in $[4,5]$ provide a very interesting extension of fuzzy sets and soft sets. $\mathfrak{f} \mathfrak{p} \mathfrak{s}$-sets provide a suitable degree of membership to both parameters and elements of the initial universe.

This is motivated by the reality that humans tend to convey their views using a simple language, which is always indeterminate, imprecise, incomplete, and inconsistent. This paper introduces the concept of fuzzy neutrosophic soft ( $\mathfrak{n} \mathfrak{n})$, in which truth-membership, falsity-membership, and indeterminacy-membership are represented. That is why $\mathfrak{f n i s -}$ sets have a sound logic to represent the rationale of human choice and are a very useful technique for finding fixed points of $\mathfrak{f n} \mathfrak{s}$-mapping and modeling uncertainties in multicriteria decision making.

Abbas et al. [8, 9] introduced the notion of soft contraction mapping based on the theory of soft elements of soft metric spaces. They studied fixed points of soft contraction

(C) The Author(s) 2018. This article is distributed under the terms of the Creative Commons Attribution 4.0 International License (http://creativecommons.org/licenses/by/4.0/), which permits unrestricted use, distribution, and reproduction in any medium, provided you give appropriate credit to the original author(s) and the source, provide a link to the Creative Commons license, and indicate if changes were made. 
mappings and obtained among others results, a soft Banach contraction principle. Akram et al. $[10,11]$ presented certain types of soft graphs based on the soft set and some novel applications of fuzzy soft graphs and m-polar fuzzy hypergraphs. Arockiarani et al. [12] presented $\mathfrak{f n}$ s-topological spaces and showed some important resolutions on it. Feng et al. studied soft sets, rough sets, fuzzy soft sets and presented an attribute analysis of information systems based on elementary soft implications. They also established an adjustable approach to fuzzy soft set-based decision making (see [13-16]). Smarandache et al. [17] suggested the idea of single valued neutrosophic set. Karaaslan [18] investigated the neu-

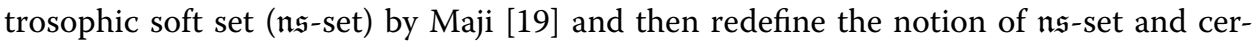
tain operations with some changes and corrections. He showed some applications based on $\mathfrak{n} \mathfrak{s - s e t s}$ to decision-making problems. Riaz et al. [20-25] established some concepts of soft sets together with soft algebra, soft $\sigma$-algebra, soft $\sigma$-ring, measurable soft set and measurable soft mappings. They established certain properties of soft metric spaces. They

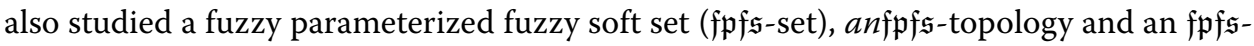
metric space and presented certain applications based on $\mathfrak{f p f s}$-sets and $\mathfrak{f p f} \mathfrak{s}$-mappings to the decision-making problems. Recently, Samet [26] defined generalized Meir-Keeler type functions and proved some coupled fixed point theorems under a generalized Meir-Keeler contractive condition. Jleli and Samet [27] introduced the concept of G-metric spaces and the fixed point existing results of contractive mappings defined on such spaces. Chen and Lin [28] obtained a soft metric version of the celebrated Meir-Keeler fixed point theorem. Wardowski [29] familiarized a concept of mapping on soft sets and determined its fixed point. A vast amount of mathematical activity has been carried out to obtain fixed points of various mappings, as studied by many authors $[8,9,26,28-31]$. In the present study, we obtain some results on fixed points of $\mathfrak{f n} \mathfrak{n}$-mappings. We introduce $\mathfrak{f n} \mathfrak{n}$-mappings by using the cartesian product with relations on fns-sets. We establish an outranking approach of $\mathfrak{f p f} \mathfrak{s}$-set to reduce energy crises. The purpose of this research is to explore some occurrences of fixed points of $\mathfrak{f n} \mathfrak{s}$-mapping and a very solid application of $\mathfrak{f} \mathfrak{p} \mathfrak{f}$-sets, which will be helpful to diminish energy crises.

Definition 1.1 $([7,17])$ Let $X$ be the universal set and a neutrosophic set $N$ is defined by $N=\left\{\left\langle\delta, T_{N}(\delta), I_{N}(\delta), F_{N}(\delta)\right\rangle, \delta \in X\right\}$, where $\left.T, I, F: X \rightarrow\right]^{-} 0,1^{+}[$and

$$
{ }^{-} 0 \leq T_{N}(\delta)+I_{N}(\delta)+F_{N}(\delta) \leq 3^{+},
$$

where $T_{N}(\delta)$ is the degree of membership, $I_{N}(\delta)$ is the degree of indeterminacy and $F_{N}(\delta)$ is the degree of falsity of elements of the given set. The neutrosophic set yields the value from real standard or non-standard subsets of $]^{-} 0,1^{+}[$. It is difficult to utilize these values in daily life science and technology problems. We consider the fuzzy neutrosophic set to be given by [12], which takes the values of the degree of membership, the degree of indeterminacy and the degree of falsity from the subset of $[0,1]$.

Definition 1.2 ([1]) A fuzzy set $F$ in $X$ is measured up by a mathematical mapping with the domain as $X$ and membership degrees in $[0,1]$. The aggregation of all fuzzy sets in universal set $X$ is symbolized by $F(X)$. 
Definition 1.3 ([12]) A fuzzy neutrosophic set (fn-set) A on the universal set $X$ is defined as $A=\left\{\left\langle\zeta, T_{A}(\zeta), I_{A}(\zeta), F_{A}(\zeta)\right\rangle, \zeta \in X\right\}$, where $T, I, F: X \rightarrow[0,1]$ and

$$
0 \leq T_{A}(\zeta)+I_{A}(\zeta)+F_{A}(\zeta) \leq 3
$$

Definition 1.4 ([2]) Let $X$ be the universal set and $R$ be the set of parameters or attributes with $A \subseteq R$, the pair $(F, A)$ is said to be a soft set over $X$, where $F$ is a mathematical function given by $F: A \rightarrow P(X)$. It can be written as

$$
(F, A)=\{(\delta, F(\delta)): \delta \in A\} .
$$

Definition 1.5 ([12]) Let $X$ be the universal set and $R$ be the set of attributes. We consider the non-empty set $A \subseteq R$. Let $\widehat{P}(X)$ denotes the assembling of all fuzzy neutrosophic sets of $X$. The aggregation $\Omega_{A}$ is called the fuzzy neutrosophic soft set (fnns-set) over $X$, where $\Omega_{A}$ is a mathematical function given by $\Omega_{A}: A \rightarrow \widehat{P}(X)$. We can write it as

$$
\Omega_{A}=\left\{\left(\delta,\left\{\left\langle\varphi, T_{A(\delta)}(\varphi), I_{A(\delta)}(\varphi), F_{A(\delta)}(\varphi)\right\rangle: \varphi \in X\right\}\right): \delta \in A\right\} .
$$

Note that if $\Omega_{A}(\delta)=\{\langle\varphi, 0,1,1\rangle: \varphi \in X\}$, the fng-element $\left(\delta, \Omega_{A}(\delta)\right)$ does not seem to be in the $\mathfrak{f n} \mathfrak{s}$-set $\Omega_{A}$. The assembling of all $\mathfrak{f n} \mathfrak{s}$-sets over $X$ is symbolized by $\mathfrak{f n} \mathfrak{s}\left(X_{R}\right)$ or $\mathfrak{f n} \mathfrak{s}(X, R)$.

We define some operations for $\mathfrak{f n} \mathfrak{s}$-sets which are different from operations of the $\mathfrak{f} \mathfrak{n} \mathfrak{s}$-sets in [12].

Definition 1.6 Let $\Omega_{A} \in \mathfrak{f n g}\left(X_{R}\right)$. If $T_{A(\zeta)}(\rho)=0, I_{A(\zeta)}(\rho)=1, F_{A(\zeta)}(\rho)=1 \forall \zeta \in R, \rho \in X$, then $\Omega_{A}$ is named as null $\mathfrak{f n} \mathfrak{n}$-set and symbolized by $\Omega_{\phi}$.

Definition 1.7 Let $\Omega_{A} \in \mathfrak{f n g}\left(X_{R}\right)$. If $T_{A(\zeta)}(\rho)=1, I_{A(\zeta)}(\rho)=0, F_{A(\zeta)}(\rho)=0 \forall \zeta \in R, \rho \in X$, then $\Omega_{A}$ is named as universal $\mathfrak{f n} \mathfrak{s}$-set and symbolized by $\Omega_{\widehat{R}}$.

Definition 1.8 Let $\Omega_{A}, \Omega_{B} \in \mathfrak{f n} \mathfrak{n}\left(X_{R}\right) . \Omega_{A}$ is said to be fnis-subset of $\Omega_{B}$, if $T_{A(\zeta)}(\rho) \leq$ $T_{B(\zeta)}(\rho), I_{A(\zeta)}(\rho) \geq I_{B(\zeta)}(\rho), F_{A(\zeta)}(\rho) \geq F_{B(\zeta)}(\rho), \forall \zeta \in R, \rho \in X$. We denote it by $\Omega_{A} \sqsubseteq \Omega_{B}$. $\Omega_{B}$ is said to be an $\mathfrak{f n s}$-superset of $\Omega_{A}$.

Definition 1.9 Let $\Omega_{A} \in \mathfrak{f n g}\left(X_{R}\right)$. Then the complement of the $\mathfrak{f n g}$-set $\Omega_{A}$ is symbolized by $\Omega_{A}^{c}$ and delineated as follows:

$$
\Omega_{A}^{c}=\left\{\left(\zeta,\left\{\left\langle\rho, F_{A(\zeta)}(\rho), 1-I_{A(\zeta)}(\rho), T_{A(\zeta)}(\rho)\right\rangle: \rho \in X\right\}\right): \zeta \in R\right\}
$$

Definition 1.10 Let $\Omega_{A}, \Omega_{B} \in \mathfrak{f n} \mathfrak{s}\left(X_{R}\right)$. Then the $\mathfrak{f n s}$-union of the $\mathfrak{f n g}$-sets $\Omega_{A}$ and $\Omega_{B}$ is symbolized by $\Omega_{A} \sqcup \Omega_{B}$ and delineated as follows:

$$
\begin{aligned}
\Omega_{A} \sqcup \Omega_{B}= & \left\{\left(\zeta,\left\{\left\langle\rho, T_{A(\zeta)}(\rho) \vee T_{B(\zeta)}(\rho), I_{A(\zeta)}(\rho) \wedge I_{B(\zeta)}(\rho), F_{A(\zeta)}(\rho) \wedge F_{B(\zeta)}(\rho)\right\rangle:\right.\right.\right. \\
& \rho \in X\}): \zeta \in R\} .
\end{aligned}
$$


Definition 1.11 Let $\Omega_{A}, \Omega_{B} \in \mathfrak{f n s}\left(X_{R}\right)$. Then the $\mathfrak{f n} \mathfrak{s}$-intersection of $\mathfrak{f n} \mathfrak{s}$-sets $\Omega_{A}$ and $\Omega_{B}$ is symbolized by $\Omega_{A} \sqcap \Omega_{B}$ and delineated as follows:

$$
\begin{aligned}
\Omega_{A} \sqcup \Omega_{B}= & \left\{\left(\zeta,\left\{\left\langle\rho, T_{A(\zeta)}(\rho) \wedge T_{B(\zeta)}(\rho), I_{A(\zeta)}(\rho) \vee I_{B(\zeta)}(\rho), F_{A(\zeta)}(\rho) \vee F_{B(\zeta)}(\rho)\right\rangle:\right.\right.\right. \\
& \rho \in X\}): \zeta \in R\} .
\end{aligned}
$$

\section{Some results on $\mathfrak{f n s}$-mapping}

In this section, we introduce the idea of fnis-mappings and present some important definitions and properties of $\mathfrak{f n} \mathfrak{s}$-mappings.

Definition 2.1 An $\mathfrak{f n} \mathfrak{n - t o p o l o g i c a l}$ space $\left(\Omega_{N}, \tau\right)$ is called $\mathfrak{f n} \mathfrak{s}$-Hausdorff space if for distinct fnis-elements $\Omega_{A}^{\zeta}, \Omega_{B}^{\zeta^{\prime}}$ of $\Omega_{N}$, there exist disjoint fnis-open sets $\Omega_{A_{1}}$ and $\Omega_{B_{1}}$ such that $\Omega_{A}^{\zeta} \in \Omega_{A_{1}}$ and $\Omega_{B}^{\zeta^{\prime}} \in \Omega_{B_{1}}$.

Proposition 2.2 Let $\left(\Omega_{N}, \tau\right)$ be an fnis-topological space. An fins-set $\Omega_{A} \sqsubseteq \Omega_{N}$ is an fnisopen if and only iffor every $\Omega_{B}^{\zeta} \in \Omega_{A}$ there exists an fni-set $\Omega_{C} \in \tau$ such that

$$
\Omega_{B}^{\zeta} \in \Omega_{C} \sqsubseteq \Omega_{A} .
$$

Proof Let $\Omega_{A} \in \tau$. Then obviously for every $\Omega_{A_{1}}^{\zeta} \in \Omega_{A}$ we have $\Omega_{A_{1}}^{\zeta} \in \Omega_{A} \sqsubseteq \Omega_{A}$. Let $\Omega_{A} \sqsubseteq$ $\Omega_{N}$ be such that for every $\Omega_{A_{1}}^{\zeta} \in \Omega_{A}$ there subsists an fns-open set $\Omega_{A_{1}}^{\zeta}$ such that $\Omega_{A_{1}}^{\zeta} \in$ $\Omega_{A_{1}^{\zeta}} \sqsubseteq \Omega_{A}$, which means that $\Omega_{A_{1}}^{\zeta}(\zeta) \in \Omega_{A_{1}^{\zeta}}\left(\zeta^{\prime}\right) \sqsubseteq \Omega_{A}\left(\zeta^{\prime}\right)$ for each $\zeta^{\prime} \in R, \Omega_{A}(\zeta)=\sqcup\left\{\Omega_{A_{1}}^{\zeta}\right.$ : $\left.\Omega_{A_{1}}^{\zeta} \in \Omega_{A}\right\} \sqsubseteq \sqcup \Omega_{A_{1}^{\zeta}}(\zeta) \sqsubseteq \Omega_{A}(\zeta)$. Therefore, $\Omega_{A}=\left\{\sqcup \Omega_{A_{1}^{\zeta}}: \zeta \in R\right\} \in \tau$.

Definition 2.3 The cartesian product of two fnis-sets $\Omega_{A}$ and $\Omega_{B}$ is defined as an $\mathfrak{f n g}$ set $\Omega_{C}=\Omega_{A} \widehat{\times} \Omega_{B}$ where $C=A \times B$ and $\Omega_{C}: C \rightarrow \mathfrak{f n} \mathfrak{s}(X, R)$ is delineated by $\Omega_{C}\left(\zeta, \zeta^{\prime}\right)=$ $\Omega_{A}(\zeta) \widehat{\times} \Omega_{B}\left(\zeta^{\prime}\right)$ for all $\left(\zeta, \zeta^{\prime}\right) \in C$, where $\Omega_{A}(\zeta) \widehat{\times} \Omega_{B}\left(\zeta^{\prime}\right)=\left\{\left\langle\rho, \min \left\{T_{A(\zeta)}(\rho), T_{B\left(\zeta^{\prime}\right)}(\rho)\right\}\right.\right.$, $\left.\left.\max \left\{I_{A(\zeta)}(\rho), I_{B\left(\zeta^{\prime}\right)}(\rho)\right\}, \max \left\{F_{A(\zeta)}(\rho), F_{B\left(\zeta^{\prime}\right)}(\rho)\right\}\right\rangle: \rho \in X\right\}$.

Example 2.4 Let $X=\left\{\rho_{1}, \rho_{2}\right\}$ and $R=\left\{\zeta_{1}, \zeta_{2}\right\}=A=B$. Define fins-sets $\Omega_{A}$ and $\Omega_{B}$ as follows: $\Omega_{A}=\left\{\left(\zeta_{1},\left\{\left\langle\rho_{1}, 0.8,0.1,0.3\right\rangle,\left\langle\rho_{2}, 0.6,0.7,0.4\right\rangle\right\}\right),\left(\zeta_{2},\left\{\left\langle\rho_{1}, 0.3,0.7,0.6\right\rangle,\left\langle\rho_{2}, 0.1,0.9\right.\right.\right.\right.$, $0.3\rangle\})\}, \quad \Omega_{B}=\left\{\left(\zeta_{1},\left\{\left\langle\rho_{1}, 0.9,0.7,0.2\right\rangle,\left\langle\rho_{2}, 0.3,0.4,0.2\right\rangle\right\}\right),\left(\zeta_{2},\left\{\left\langle\rho_{1}, 0.1,0.3,0.6\right\rangle,\left\langle\rho_{2}, 0.7,0.3\right.\right.\right.\right.$, $0.9\rangle\})$ \}. We use $\mathfrak{f n}$ s-sets in tabular form to make the calculations easy.

and

\begin{tabular}{l|ll}
$\Omega_{A}$ & $\zeta_{1}$ & $\zeta_{2}$ \\
\hline$\rho_{1}$ & $(0.8,0.1,0.3)$ & $(0.3,0.7,0.6)$ \\
$\rho_{2}$ & $(0.6,0.7,0.4)$ & $(0.1,0.9,0.3)$
\end{tabular}

\begin{tabular}{l|ll}
$\Omega_{B}$ & $\zeta_{1}$ & $\zeta_{2}$ \\
\hline$\rho_{1}$ & $(0.9,0.7,0.2)$ & $(0.1,0.3,0.6)$ \\
$\rho_{2}$ & $(0.3,0.4,0.2)$ & $(0.7,0.3,0.9)$
\end{tabular}

Then $\Omega_{A} \widehat{\times} \Omega_{B}=\Omega_{C}$ where $C=A \times B$ and $\Omega_{A}(\zeta) \widehat{\times} \Omega_{B}\left(\zeta^{\prime}\right)$ calculated as

\begin{tabular}{l|llll}
$\Omega_{C}$ & $\left(\zeta_{1}, \zeta_{1}\right)$ & $\left(\zeta_{1}, \zeta_{2}\right)$ & $\left(\zeta_{2}, \zeta_{1}\right)$ & $\left(\zeta_{2}, \zeta_{2}\right)$ \\
\hline$\rho_{1}$ & $(0.8,0.7,0.3)$ & $(0.1,0.3,0.6)$ & $(0.3,0.7,0.6)$ & $(0.1,0.7,0.6)$ \\
$\rho_{2}$ & $(0.3,0.7,0.4)$ & $(0.6,0.7,0.9)$ & $(0.1,0.9,0.3)$ & $(0.1,0.9,0.9)$
\end{tabular}

Definition 2.5 Let $\Omega_{A}$ and $\Omega_{B}$ be $\mathfrak{f n s}$-sets in $\mathfrak{f n s}(X, R)$. An $\mathfrak{f n} \mathfrak{s - s e t} \mathbb{R}$ is said to be an $\mathfrak{f n s}$ relation from $\Omega_{A}$ to $\Omega_{B}$ if $\mathbb{R}=\Omega_{D}$ where $D \subseteq C=A \times B$ and $\Omega_{D}: D \rightarrow \mathfrak{f n g}(X, R)$ on $D$. 
Example 2.6 Let $\Omega_{A}$ and $\Omega_{B}$ be fnis-sets in Example 2.4. Then $\mathbb{R}=\left\{\Omega_{A}\left(\zeta_{1}\right) \widehat{\times} \Omega_{B}\left(\zeta_{1}\right)\right.$, $\left.\Omega_{A}\left(\zeta_{1}\right) \widehat{\times} \Omega_{B}\left(\zeta_{2}\right), \Omega_{A}\left(\zeta_{2}\right) \widehat{\times} \Omega_{B}\left(\zeta_{1}\right)\right\}$ is a fnis-relation from $\Omega_{A}$ to $\Omega_{B}$ which itself is an fns-set with $\left\{\left(\zeta_{1}, \zeta_{1}\right),\left(\zeta_{1}, \zeta_{2}\right),\left(\zeta_{2}, \zeta_{1}\right)\right\}$ as a set of parameters. By $\Omega_{A} \mathbb{R} \Omega_{B}$ we mean that $\Omega_{A}\left(\zeta_{1}\right) \widehat{\times} \Omega_{B}\left(\zeta_{2}\right) \in \mathbb{R}$.

In the next definition, we introduce an $\mathfrak{f n} \mathfrak{s}$-mapping.

Definition 2.7 Let $\Omega_{A}$ and $\Omega_{B}$ be fins-sets in $\mathfrak{f n s}(X, R)$. An fngs-relation $\Upsilon$ from $\Omega_{A}$ to $\Omega_{B}$ is said to be an fins-mapping from $\Omega_{A}$ to $\Omega_{B}$ symbolized by $\Upsilon: \Omega_{A} \rightarrow \Omega_{B}$ if these properties are gratified. $C_{1}$ : For every fns-element $\Omega_{A_{1}}^{\zeta} \in \Omega_{A}$, there exists only one finselement $\Omega_{B_{1}}^{\zeta} \in \Omega_{B}$ such that

$$
\Upsilon\left(\Omega_{A_{1}}^{\zeta}\right)=\Omega_{B_{1}}^{\zeta}
$$

$C_{2}$ : For each empty fnis-element $\Omega_{\phi}^{\zeta} \in \Omega_{A}, \Upsilon\left(\Omega_{\phi}^{\zeta}\right)$ is an empty fins-element for $\Omega_{B}$.

Definition 2.8 Let $\Omega_{A}$ and $\Omega_{B}$ be fns-sets in $\mathfrak{f n s}(X, R)$ and $\Upsilon: \Omega_{A} \rightarrow \Omega_{B}$ be an $\mathfrak{f n s}$ mapping. The image of $\Omega_{C} \sqsubseteq \Omega_{A}$ under fnis-mapping $\Upsilon$ is the fns-set $\Upsilon\left(\Omega_{C}\right)$ defined by $\Upsilon\left(\Omega_{C}\right)=\left\{\bigsqcup_{\Omega_{A_{1}}^{\zeta} \in \Omega_{C}} \Upsilon\left(\Omega_{A_{1}}^{\zeta}\right): \zeta \in R\right\}$. It is obvious that $\Upsilon\left(\Omega_{\phi}\right)=\Omega_{\phi}$ for every finsmapping $\Upsilon$.

Definition 2.9 Let $\Omega_{A}$ and $\Omega_{B}$ be fngs-sets in $\mathfrak{f n s}(X, R)$ and $\Upsilon: \Omega_{A} \rightarrow \Omega_{B}$ be an $\mathfrak{f n s}-$ mapping. The inverse image of $\Omega_{D} \sqsubseteq \Omega_{B}$ under fins-mapping $\Upsilon$ is the fnis-set symbolized by $\Upsilon^{-1}\left(\Omega_{D}\right)$ and delineated as $\Upsilon^{-1}\left(\Omega_{D}\right)=\left\{\left\{\bigsqcup_{\Omega_{A_{1}}^{\zeta} \in \Omega_{A}} \Upsilon\left(\Omega_{A_{1}}^{\zeta}\right): \zeta \in R\right\}: \Upsilon\left(\Omega_{A_{1}}^{\zeta}\right) \in\right.$ $\Omega_{D}$ for each $\left.\zeta \in R\right\}$.

Example 2.10 Let $\Omega_{A}$ and $\Omega_{B}$ be given in Example 2.4. Define $\Upsilon$ as $\Upsilon\left(\Omega_{A_{1}}^{\zeta}\right)=\widehat{\Omega}_{B_{1}}^{\zeta}$ for each $\zeta \in R$, where $\widehat{\Omega}_{B_{1}}^{\zeta}$ is the greatest fnis-element for every attribute $\zeta \in R$, that is, if $\Omega_{B_{1}}^{\zeta}$ is an arbitrary fns-element in $\Omega_{B}$ then $\Omega_{B_{1}}^{\zeta} \sqsubseteq \widehat{\Omega}_{B_{1}}^{\zeta}$. So, $\Upsilon\left(\Omega_{A_{1}}^{\zeta_{1}}\right)=\widehat{\Omega}_{B_{1}}^{\zeta_{1}}=$ $\left\{\left\langle\rho_{1}, 0.9,0.7,0.2\right\rangle,\left\langle\rho_{2}, 0.3,0.4,0.2\right\rangle\right\}$ for all $\Omega_{A}^{\zeta_{1}} \in \Omega_{A}$ and $\Upsilon\left(\Omega_{A_{1}}^{\zeta_{2}}\right)=\widehat{\Omega}_{B_{1}}^{\zeta_{2}}=\left\{\left\langle\rho_{1}, 0.1,0.3,0.6\right\rangle\right.$, $\left.\left\langle\rho_{2}, 0.7,0.3,0.9\right\rangle\right\}$ for all $\Omega_{A}^{\zeta_{2}} \in \Omega_{A}$. Moreover, $\Upsilon(\Omega)=\left\{\bigsqcup_{\Omega_{A}^{\zeta} \in \Omega_{C}} \Upsilon\left(\Omega_{A}^{\zeta}\right): \zeta \in R\right\}=$ $\left\{\left\{\bigsqcup_{\Omega_{A}^{\zeta_{1}} \in \Omega_{C}} \Upsilon\left(\Omega_{A}^{\zeta_{1}}\right)\right\},\left\{\bigsqcup_{\Omega_{A}^{\zeta_{2}} \in \Omega_{C}} \Upsilon\left(\Omega_{A}^{\zeta_{2}}\right)\right\}\right\}=\left\{\widehat{\Omega}_{B}^{\zeta_{1}}, \widehat{\Omega}_{B}^{\zeta_{2}}\right\}=\Omega_{B}$

Definition 2.11 Let $\left(\Omega_{A}, \tau\right)$ be an $\mathfrak{f n} \mathfrak{s - t o p o l o g i c a l}$ space and $\Omega_{B} \sqsubseteq \Omega_{A}$. An fins-open cover for $\Omega_{B}$ is an assembling of $\mathfrak{f n} \mathfrak{s}-$ open sets $\left\{\Omega_{\alpha}\right\}_{\alpha \in I} \sqsubseteq \tau$ whose $\mathfrak{f n} \mathfrak{s}$-union carries $\Omega_{B}$.

Definition 2.12 An $\mathfrak{f n} \mathfrak{s - t o p o l o g i c a l}$ space $\left(\Omega_{A}, \tau\right)$ is $\mathfrak{f n} \mathfrak{i}$-compact if for every $\mathfrak{f n} \mathfrak{i}$-open cover $\left\{\Omega_{\alpha}\right\}_{\alpha \in I}$ of $\Omega_{B}$ there subsists $\alpha_{1}, \alpha_{2}, \alpha_{3}, \ldots, \alpha_{k} \in I, k \in N$ such that $\Omega_{B} \sqsubseteq \bigsqcup_{n=1}^{k} \Omega_{\alpha_{n}}$.

Definition 2.13 Let $\left(\Omega_{A}, \tau\right),\left(\Omega_{B}, \tau^{\prime}\right)$ be fins-topological spaces and $\Upsilon: \Omega_{A} \rightarrow \Omega_{B}$ an finsmapping. Then $\Upsilon$ be an fins-continuous function if for every $\Omega_{B_{1}} \in \tau^{\prime}, \Upsilon^{-1}\left(\Omega_{B_{1}}\right) \in \tau$, that is, the inverse image of an $\mathfrak{f n g}$-open set is an $\mathfrak{f n} \mathfrak{n}$-open set. An fins-set $\Omega_{C} \sqsubseteq \Omega_{A}$ is an $\mathfrak{f n g}$ compact in $\left(\Omega_{A}, \tau\right)$ if the $\mathfrak{f n s}$-topological space $\left(\Omega_{C}, \tau_{C}\right)$ is $\mathfrak{f n} \mathfrak{s}$-compact.

Proposition 2.14 Let $\left(\Omega_{A}, \tau\right)$ be an fnis-compact topological space and $\Upsilon: \Omega_{A} \rightarrow \Omega_{A}$ an fns-continuous function. Then $\Upsilon\left(\Omega_{A}\right)$ is an fnis-compact set in $\left(\Omega_{A}, \tau\right)$. 
Proof Consider that $\Upsilon\left(\Omega_{A}\right) \sqsubseteq \bigsqcup_{i} \Omega_{i}$, where $\left\{\Omega_{i}\right\}$ is an assembling of $\mathfrak{f n} \mathfrak{s - o p e n}$ sets in $\Omega_{A}$. Then taking the pre-image, we have $\Omega_{A} \sqsubseteq \Upsilon^{-1}\left(\bigsqcup_{i} \Omega_{i}\right)$. As $\Upsilon^{-1}\left(\Omega_{i}\right)$ is fins-open in $\Psi_{i}$ so there must exists fnis-open $\Psi_{i} \sqsubseteq \Upsilon\left(\Omega_{A}\right)$ such that $\Upsilon^{-1}\left(\Omega_{i}\right)=\Psi_{i} \sqcap \Omega_{A}$. So $\Omega_{A} \sqsubseteq \bigsqcup_{i}\left(\Psi_{i} \sqcap\right.$ $\left.\Omega_{A}\right)$ implies that $\Omega_{A} \sqsubseteq \bigsqcup_{i} \Psi_{i}$. Since $\Omega_{A}$ is an fng-compact set, there exist $i_{1}, i_{2}, \ldots, i_{\alpha}$ such that $\Omega_{A} \sqsubseteq \bigsqcup_{n=1}^{\alpha} \Psi_{i_{n}}$. Thus $\Omega_{A}=\bigsqcup_{i}\left(\Psi_{i} \sqcap \Omega_{A}\right)=\bigsqcup_{n=1}^{\alpha} \Upsilon^{-1}\left(\Omega_{i_{n}}\right)$ this implies that $\Upsilon\left(\Omega_{A}\right) \sqsubseteq$ $\bigsqcup_{n=1}^{\alpha}\left(\Omega_{i_{n}}\right)$. Hence $\Upsilon\left(\Omega_{A}\right)$ is $\mathfrak{f n} \mathfrak{s}$-compact.

\section{Fixed points of $\mathfrak{f n s}$-mappings}

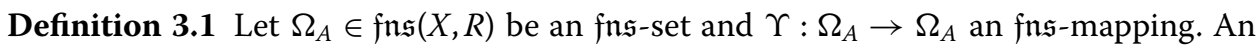
fns-element $\Omega_{A}^{\zeta} \in \Omega_{A}$ is said to be a fixed point of $\Upsilon$ if $\Upsilon\left(\Omega_{A}^{\zeta}\right)=\Omega_{A}^{\zeta}$.

Example 3.2 If $\Upsilon: \Omega_{A} \rightarrow \Omega_{A}$ is an identity mapping, then every fnis-element of $\Omega_{A}$ is a fixed point.

Proposition 3.3 Let $\left(\Omega_{A}, \tau\right)$ be an fnis-compact topological space and $\left\{\Omega_{A_{\alpha}}: \alpha \in N\right\}$ a countable assembling of $\mathfrak{f n} \mathfrak{s - s u b s e t s}$ of $\Omega_{A}$ obeying:

(a) $\Omega_{A_{\alpha}} \neq \Omega_{\phi}$ for every $\alpha \in N$,

(b) $\Omega_{A_{\alpha}}$ is fins-closed for each $\alpha \in N$,

(c) $\Omega_{A_{\alpha+1}} \sqsubseteq \Omega_{A_{\alpha}}$ for each $\alpha \in N$. Then $\prod_{\alpha \in N} \Omega_{A_{\alpha}} \neq \Omega_{\phi}$.

Proof Suppose on contrary that $\prod_{\alpha \in N} \Omega_{A_{\alpha}}=\Omega_{\phi}$. We know that $\left(\prod_{\alpha \in N} \Omega_{A_{\alpha}}\right)^{c}=$ $\bigsqcup_{\alpha \in N}\left(\Omega_{A_{\alpha}}\right)^{c}$. From (b), $\left(\Omega_{A_{\alpha}}\right)^{c}$ is fnis-open set for each $\alpha \in N$. Hence $\Omega_{A_{\alpha}} \sqsubseteq \Omega_{\widetilde{R}}=\left(\Omega_{\phi}\right)^{c}=$ $\left(\prod_{\alpha \in N} \Omega_{A_{\alpha}}\right)^{c}=\bigsqcup_{\alpha \in N}\left(\Omega_{A_{\alpha}}\right)^{c}$. As $\Omega_{A}$ is fns-compact, there exists $i_{1}, i_{2}, \ldots, i_{k} \in N, i_{1}<$ $i_{2}<\cdots<i_{k}, k \in N$ such that $\Omega_{A} \sqsubseteq \Omega_{A_{i_{1}}}^{c} \sqcup \Omega_{A_{i_{2}}}^{c} \sqcup \cdots \sqcup \Omega_{A_{i_{k}}}^{c}$. Hence from (c) we have $\Omega_{A_{i_{k}}} \sqsubseteq \Omega_{A} \sqsubseteq\left(\Omega_{A_{i_{1}}} \sqcap \Omega_{A_{i_{2}}} \sqcap \cdots \sqcap \Omega_{A_{i_{k}}}\right)^{c}=\left(\Omega_{A_{i_{k}}}\right)^{c}=\Omega_{\widetilde{R}} / \Omega_{A_{i_{k}}}$, which is not possible by (a).

Example 3.4 Let $\left(\Omega_{A}, \tau\right)$ be an $\mathfrak{f n} \mathfrak{s - t o p o l o g i c a l}$ space and $\Omega_{A}$ given by $\Omega_{A}=\left\{\left(\zeta_{1},\left\{\left\langle\rho_{1}, 0.7\right.\right.\right.\right.$, $\left.\left.\left.0.2,0.1\rangle,\left\langle\rho_{2}, 0.5,0.3,0.4\right\rangle\right\}\right),\left(\zeta_{2},\left\{\left\langle\rho_{1}, 0.9,0.1,0.3\right\rangle,\left\langle\rho_{2}, 0.6,0.4,0.2\right\rangle\right\}\right)\right\}$. The tabular form is given by

\begin{tabular}{c|cc}
$\Omega_{A}$ & $\zeta_{1}$ & $\zeta_{2}$ \\
\hline$\rho_{1}$ & $(0.7,0.2,0.1)$ & $(0.9,0.1,0.3)$ \\
$\rho_{2}$ & $(0.5,0.3,0.4)$ & $(0.6,0.4,0.2)$
\end{tabular}

Let two fnis-subsets of $\Omega_{A}$ namely $\Omega_{A_{1}}$ and $\Omega_{A_{2}}$ be given as $\Omega_{A_{1}}=\left\{\left(\zeta_{1},\left\{\left\langle\rho_{1}, 0.6,0.3,0.4\right\rangle\right.\right.\right.$, $\left.\left.\left.\left\langle\rho_{2}, 0.2,0.9,0.7\right\rangle\right\}\right),\left(\zeta_{2},\left\{\left\langle\rho_{1}, 0.7,0.5,0.6\right\rangle,\left\langle\rho_{2}, 0.3,0.6,0.3\right\rangle\right\}\right)\right\} \Omega_{A_{2}}=\left\{\left(\zeta_{1},\left\{\left\langle\rho_{1}, 0.7,0.4,0.5\right\rangle\right.\right.\right.$, $\left.\left.\left.\left\langle\rho_{2}, 0.3,0.9,0.8\right\rangle\right\}\right),\left(\zeta_{2},\left\{\left\langle\rho_{1}, 0.8,0.6,0.7\right\rangle,\left\langle\rho_{2}, 0.5,0.7,0.4\right\rangle\right\}\right)\right\}$ The tabular forms of these sets are given below:

and

\begin{tabular}{c|cc}
$\Omega_{A_{1}}$ & $\zeta_{1}$ & $\zeta_{2}$ \\
\hline$\rho_{1}$ & $(0.6,0.3,0.4)$ & $(0.7,0.5,0.6)$ \\
$\rho_{2}$ & $(0.2,0.9,0.7)$ & $(0.3,0.6,0.3)$
\end{tabular}

\begin{tabular}{c|cc}
$\Omega_{A_{2}}$ & $\zeta_{1}$ & $\zeta_{2}$ \\
\hline$\rho_{1}$ & $(0.7,0.4,0.5)$ & $(0.8,0.6,0.7)$ \\
$\rho_{2}$ & $(0.3,0.9,0.8)$ & $(0.5,0.7,0.4)$
\end{tabular}

These sets gratify the properties of Proposition 3.3. Moreover, $\Omega_{A_{1}} \sqsubseteq \Omega_{A_{2}}$ and $\prod_{\alpha=1}^{2} \Omega_{A_{\alpha}}=$ $\Omega_{A_{1}} \neq \Omega_{\phi}$. 
Proposition 3.5 Let $\left(\Omega_{A}, \tau\right)$ be an fnis-topological space and $\Upsilon: \Omega_{A} \rightarrow \Omega_{A}$ be an finsmapping such that for every non-empty fni-element $\Omega_{A}^{\zeta} \in \Omega_{A}, \Upsilon\left(\Omega_{A}^{\zeta}\right)$ is a non-empty $\mathfrak{f n g}$ element of $\Omega_{A}$. If $\prod_{\alpha \in N} \Upsilon^{\alpha}\left(\Omega_{A}\right)$ contains only one non-empty fins-element $\Omega_{A}^{\zeta} \in \Omega_{A}$, then $\Omega_{A}^{\zeta}$ is a unique fixed point of $\Upsilon$.

Proof Observe that $\Upsilon^{\alpha}\left(\Omega_{A}\right) \sqsubseteq \Upsilon^{\alpha-1}\left(\Omega_{A}\right)$ for each $\alpha \in N$. Let $\Omega_{A}^{\zeta}$ be an fns-element of $\Omega_{A}$ such that $\Omega_{A}^{\zeta} \in \prod_{\alpha \in N} \Upsilon^{\alpha}\left(\Omega_{A}\right)$. That is, $\Omega_{A}^{\zeta} \sqsubseteq \prod_{\alpha \in N} \Upsilon^{\alpha}\left(\Omega_{A}\right)$. Consequently, $\Upsilon\left(\Omega_{A}^{\zeta}\right) \sqsubseteq$ $\Upsilon\left(\prod_{\alpha \in N} \Upsilon^{\alpha}\left(\Omega_{A}\right)\right) \sqsubseteq \prod_{\alpha \in N} \Upsilon^{\alpha+1}\left(\Omega_{A}\right) \sqsubseteq \prod_{\alpha \in N} \Upsilon^{\alpha}\left(\Omega_{A}\right)=\Omega_{A}^{\zeta}$. Since $\Upsilon\left(\Omega_{A}^{\zeta}\right)$ is a non-empty fni-element of $\Omega_{A}$, we get $\Upsilon\left(\Omega_{A}^{\zeta}\right)=\Omega_{A}^{\zeta}$.

Example 3.6 Let $\left(\Omega_{A}, \tau\right)$ be an fnis-topological space and define $\Upsilon: \Omega_{A} \rightarrow \Omega_{A}$ as $\Upsilon\left(\Omega_{A}^{\zeta}\right)=$ $\widehat{\Omega}_{A}^{\zeta}$ for all $\Omega_{A}^{\zeta} \in \Omega_{A}$, where $\Omega_{A} \neq \Omega_{\phi}$ and $\widehat{\Omega}_{A}^{\zeta}$ represents the largest fnis-element of $\Omega_{A}$ or equivalently $\Omega_{A}^{\zeta} \sqsubseteq \widehat{\Omega}_{A}^{\zeta}$ for each fnis-element $\Omega_{A}^{\zeta} \in \Omega_{A}$. Then $\prod_{\alpha \in N} \Upsilon^{\alpha}\left(\Omega_{A}\right)$ carries only one non-empty fnis-element $\widehat{\Omega}_{A}^{\zeta}$. Thus $\widehat{\Omega}_{A}^{\zeta}$ is a unique fixed point of $\Upsilon$.

Proposition 3.7 Let $\left(\Omega_{A}, \tau\right)$ be an $\mathfrak{f n} \mathfrak{s}$-Hausdorff topological space. Then each $\mathfrak{f n} \mathfrak{s -}$ compact set in $\Omega_{A}$ is fins-closed in $\Omega_{A}$.

Proof Let $\Psi_{B}$ be an $\mathfrak{f n} \mathfrak{s}$-compact set in $\left(\Omega_{A}, \tau\right)$, we here show that $\Psi_{B}$ is $\mathfrak{f n} \mathfrak{n - c l o s e d}$, that is, $\Psi_{B}^{c}$ is fng-open. Let $\Omega_{A}^{\zeta} \in \Psi_{B}^{c}$, for every $\Omega_{A}^{\zeta^{\prime}} \in \Psi_{B}$, let $U_{\alpha}, V_{\alpha} \in \tau$ be such that $U_{\alpha} \sqcap V_{\alpha}=\Omega_{\phi}$ and $\Omega_{A}^{\zeta} \in U_{\alpha}, \Omega_{A}^{\zeta^{\prime}} \in V_{\alpha}$ where $\alpha \in I$. Since $\Psi_{B}$ is fins-compact, there exist $\Omega_{A}^{\zeta^{\prime}}, \Omega_{A}^{\zeta^{\prime}}, \ldots, \Omega_{A}^{\zeta^{\prime}} \in$ $\Psi_{B}$ such that $\Psi_{B} \sqsubseteq V_{\alpha_{1}} \sqcup V_{\alpha_{2}} \sqcup \cdots \sqcup V_{\alpha_{k}}$. Denote $U=U_{\alpha_{1}} \sqcup U_{\alpha_{2}} \sqcup \cdots \sqcup U_{\alpha_{k}}$ and $V=V_{\alpha_{1}} \sqcup$ $V_{\alpha_{2}} \sqcup \cdots \sqcup V_{\alpha_{k}}$. Then $\Omega_{A}^{\zeta} \in U \in \tau, U \sqcap V=\Omega_{\phi}$, which implies that $\Omega_{A}^{\zeta} \in U \sqsubseteq \Psi_{B}^{c}$. Thus $\Psi_{B}$ is $\mathfrak{f n} \mathfrak{s - c l o s e d}$.

Theorem 3.8 Let $\left(\Omega_{A}, \tau\right)$ be an fnis-Hausdorff topological space and $\Upsilon: \Omega_{A} \rightarrow \Omega_{A}$ an fniscontinuous function such that:

(a) for every non-empty fni-element $\Omega_{A}^{\zeta} \in \Psi_{B}, \Upsilon\left(\Omega_{A}^{\zeta}\right)$ is a non-empty fni-element of $\Psi_{B}$,

(b) for every fns-closed set $\Psi_{C} \sqsubseteq \Psi_{B}$ if $\Upsilon\left(\Psi_{C}\right)=\Psi_{C}$ then $\Psi_{C}$ contains only one non-empty fni-element of $\Psi_{B}$. Then there subsists a unique non-empty fng-element $\Omega_{A}^{\zeta} \in \Psi_{B}$ such that $\Upsilon\left(\Omega_{A}^{\zeta}\right)=\Omega_{A}^{\zeta}$.

Proof Suppose an assembling of $\mathfrak{f n s}$-subsets of $\Psi_{B}$ of the form $Z_{1}=\Upsilon\left(\Psi_{B}\right), Z_{2}=\Upsilon\left(Z_{1}\right)$, $Z_{3}=\Upsilon\left(Z_{2}\right), \ldots, Z_{\alpha}=\Upsilon\left(Z_{\alpha-1}\right)=\Upsilon^{\alpha}\left(\Psi_{B}\right)$ for $\alpha \in N$. It is clear that $Z_{\alpha} \sqsubseteq Z_{\alpha-1}$ for every $\alpha \in N$. By Proposition 3.7, for every $\alpha \in N, Z_{\alpha}$ is $\mathfrak{f n} \mathfrak{s - c l o s e d}$. Using Proposition 3.3, it is clear that an $\mathfrak{f n s}$-set $\Psi_{D}$ of the form $\Psi_{D}=\prod_{\alpha \in N} Z_{\alpha}$ is non-empty. Observe that $\Upsilon\left(\Psi_{D}\right)=\Upsilon\left(\prod_{\alpha \in N} \Upsilon^{\alpha}\left(\Psi_{B}\right)\right) \sqsubseteq \prod_{\alpha \in N} \Upsilon^{\alpha+1}\left(\Psi_{B}\right) \sqsubseteq \prod_{\alpha \in N} \Upsilon^{\alpha}\left(\Psi_{B}\right)=\Psi_{D}$. Next we will prove that $\Psi_{D}=\Upsilon\left(\Psi_{D}\right)$. For this purpose, consider that there subsists $\Omega_{A}^{\zeta} \in \Psi_{D}$ such that $\Omega_{A}^{\zeta}$ is not an fns-element of $\Upsilon\left(\Psi_{D}\right)$. Put $F_{\alpha}=\Upsilon^{-1}\left(\Omega_{A}^{\zeta}\right) \sqcap Z_{\alpha}$. Let us observe that $F_{\alpha} \neq \Omega_{\phi}$ and $F_{\alpha} \sqsubseteq F_{\alpha-1}$ for each $\alpha \in N$. By Proposition 3.3, there exists a non-empty fnis-element $\Omega_{A}^{\zeta^{\prime}} \in$ $\Upsilon^{-1}\left(\Omega_{A}^{\zeta}\right) \sqcap \Psi_{D}$ and thus $\Omega_{A}^{\zeta}=\Upsilon\left(\Omega_{A}^{\zeta^{\prime}}\right) \in \Upsilon\left(\Psi_{D}\right)$, a contradiction. Therefore, $\Upsilon\left(\Psi_{D}\right)=\Psi_{D}$. Hence the result follows using Proposition 3.3.

\section{An application of the fns-set to multi-criteria decision making}

Definition 4.1 ([32]) We have a matrix, where rows represent the person names $p_{1}, p_{2}, p_{3}$, $\ldots, p_{n}$ and columns represent the parameters $q_{1}, q_{2}, q_{3}, \ldots, q_{m}$. The entries $\varepsilon_{\alpha \beta}$ are designed by $\varepsilon_{\alpha \beta}=a+b-c$, where a is the number premeditated as how many times $T_{p_{\alpha}}\left(q_{\beta}\right)$ exceeds 
Table $1 \mathfrak{f n s}$-data

\begin{tabular}{llllllll}
\hline $\mathbf{X}$ & $\begin{array}{l}\text { Over } \\
\text { population }\end{array}$ & $\begin{array}{l}\text { Wastage of } \\
\text { energy }\end{array}$ & $\begin{array}{l}\text { Poor infras- } \\
\text { tructure }\end{array}$ & $\begin{array}{l}\text { Poor distri- } \\
\text { bution } \\
\text { system }\end{array}$ & $\begin{array}{l}\text { Major } \\
\text { accidents } \\
\text { and natural } \\
\text { calamities }\end{array}$ & $\begin{array}{l}\text { Wars and } \\
\text { attacks }\end{array}$ & $\begin{array}{l}\text { Over con- } \\
\text { sumption }\end{array}$ \\
\hline$S_{1}$ & $(0.7,0.1,0.3)$ & $(0.8,0.2,0.3)$ & $(0.9,0.2,0.4)$ & $(0.9,0.2,0.1)$ & $(0.8,0.1,0.2)$ & $(0.8,0.3,0.3)$ & $(0.9,0.2,0.2)$ \\
$\varsigma_{2}$ & $(0.3,0.5,0.7)$ & $(0.4,0.5,0.1)$ & $(0.6,0.1,0.4)$ & $(0.4,0.3,0.1)$ & $(0.6,0.1,0.4)$ & $(0.5,0.2,0.1)$ & $(0.7,0.1,0.1)$ \\
$\varsigma_{3}$ & $(0.4,0.2,0.1)$ & $(0.7,0.3,0.2)$ & $(0.6,0.5,0.1)$ & $(0.6,0.1,0.2)$ & $(0.8,0.7,0.6)$ & $(0.6,0.5,0.3)$ & $(0.4,0.1,0.1)$ \\
$\varsigma_{4}$ & $(0.6,0.2,0.3)$ & $(0.5,0.4,0.4)$ & $(0.6,0.5,0.5)$ & $(0.5,0.2,0.1)$ & $(0.4,0.1,0.2)$ & $(0.5,0.1,0.2)$ & $(0.2,0.1,0.1)$ \\
$\varsigma_{5}$ & $(0.9,0.1,0.1)$ & $(0.8,0.2,0.2)$ & $(0.7,0.1,0.2)$ & $(0.9,0.1,0.2)$ & $(0.9,0.2,0.1)$ & $(0.8,0.1,0.3)$ & $(0.7,0.2,0.2)$ \\
$\varsigma_{6}$ & $(0.7,0.2,0.3)$ & $(0.8,0.2,0.2)$ & $(0.9,0.1,0.2)$ & $(0.6,0.1,0.2)$ & $(0.6,0.2,0.3)$ & $(0.6,0.1,0.4)$ & $(0.7,0.2,0.5)$ \\
$\varsigma_{7}$ & $(0.6,0.1,0.3)$ & $(0.7,0.1,0.2)$ & $(0.6,0.1,0.1)$ & $(0.6,0.3,0.4)$ & $(0.5,0.1,0.2)$ & $(0.6,0.2,0.1)$ & $(0.8,0.1,0.1)$ \\
$\varsigma_{8}$ & $(0.4,0.3,0.2)$ & $(0.5,0.1,0.4)$ & $(0.3,0.1,0.1)$ & $(0.4,0.3,0.2)$ & $(0.5,0.2,0.4)$ & $(0.4,0.2,0.3)$ & $(0.5,0.5,0.4)$ \\
\hline
\end{tabular}

or equals $T_{p_{\gamma}}\left(q_{\beta}\right)$ for $p_{\alpha} \neq p_{\gamma} \forall p_{\gamma} \in X$, $\mathrm{b}$ is the number premeditated as how many times $I_{p_{\alpha}}\left(q_{\beta}\right)$ exceeds or equals $I_{p_{\gamma}}\left(q_{\beta}\right)$ for $p_{\alpha} \neq p_{\gamma} \forall p_{\gamma} \in X$ and $\mathrm{c}$ is the number premeditated as how many times $F_{p_{\alpha}}\left(q_{\beta}\right)$ exceeds or equals $F_{p_{\gamma}}\left(q_{\beta}\right)$ for $p_{\alpha} \neq p_{\gamma} \forall p_{\gamma} \in X$.

Application: An energy disaster is any substantial blockage in the provision of energy resources to an economy. Energy is the supreme significant source of national power. Any country cannot attain financial success and military strength without passable sources of energy. Let $X=\left\{\varsigma_{1}, \varsigma_{2}, \varsigma_{3}, \ldots, \varsigma_{m}\right\}$ be the universal set where the $\varsigma_{j}, j=1,2,3, \ldots, m$ represent the possible solutions to reduce energy crises and $\mathfrak{R}=\left\{\varrho_{1}, \varrho_{2}, \varrho_{3}, \ldots, \varrho_{n}\right\}$ be the assembling of alternatives or causes which increase this problem by the criteria of the fnis-set. Here $\varrho_{i}, i=1,2,3, \ldots, n$ is for the alternatives or causes for the criteria of the fnis-set. Now we demonstrate an algorithm for most suitable choice of an object.

Algorithm:

Input:

step 1: Construct the table of given data in the form of the fnis-sets.

Output:

step 2: Calculate the average values by using fins-average operator,

$$
\mathfrak{A}_{k}=\left(\frac{\sum_{i=1}^{n} T_{i j}}{n}, \frac{\sum_{i=1}^{n} I_{i j}}{n}, \frac{\sum_{i=1}^{n} F_{i j}}{n}\right)
$$

for each $i=1,2,3, \ldots, n$ and $j=1,2,3, \ldots, m$.

step 3: Compute the comparison table $C_{T}$ by following Definition 4.1.

step 4: The maximum scored value should be more preferable from set $X$ by using $\max _{j}\left(C_{j}\right)$.

Example 4.2 Suppose that a country is facing energy crisis problems. The authorities want to control this problem and create possible solutions to relieve it. Agreeing to a survey, experts find some main reasons of energy crises. They listed some possible solutions to curb this problem. We construct here a $\mathfrak{f n} \mathfrak{s - m o d e l}$ to find the order-wise possible solutions to curb this problem. The fns-information about the causes and possible solutions is given in Table 1.

The set of possible solutions is represented by $X=\left\{\varsigma_{1}, \varsigma_{2}, \varsigma_{3}, \varsigma_{4}, \varsigma_{5}, \varsigma_{6}, \varsigma_{7}, \varsigma_{8}\right\}$, where:

$\varsigma_{1}=$ Buy energy efficient products;

$\varsigma_{2}=$ Lighting controls;

$\varsigma_{3}=$ Energy simulation; 
Table $2 \mathfrak{f n s}$-average table

\begin{tabular}{ll}
\hline $\mathbf{X}$ & Average values \\
\hline$\varsigma_{1}$ & $(0.828,0.185,0.257)$ \\
$\varsigma_{2}$ & $(0.5,0.257,0.271)$ \\
$\varsigma_{3}$ & $(0.585,0.342,0.228)$ \\
$\varsigma_{4}$ & $(0.471,0.228,0.257)$ \\
$\varsigma_{5}$ & $(0.814,0.142,0.185)$ \\
$\varsigma_{6}$ & $(0.7,0.157,0.3)$ \\
$\varsigma_{7}$ & $(0.628,0.142,0.2)$ \\
$\varsigma_{8}$ & $(0.428,0.242,0.285)$ \\
\hline
\end{tabular}

Table $3 \mathfrak{f n s}$-comparison table

\begin{tabular}{ll}
\hline $\boldsymbol{C}_{\boldsymbol{T}}$ & Comparison values \\
\hline $\boldsymbol{S}_{1}$ & $7+3-4=6$ \\
$\boldsymbol{S}_{2}$ & $2+6-4=4$ \\
$\boldsymbol{S}_{3}$ & $3+7-2=8$ \\
$\boldsymbol{S}_{4}$ & $1+4-4=1$ \\
$\boldsymbol{S}_{5}$ & $6+1-0=7$ \\
$\boldsymbol{S}_{6}$ & $6+2-7=1$ \\
$\boldsymbol{S}_{7}$ & $4+1-1=4$ \\
$\boldsymbol{S}_{8}$ & $0+5-6=-1$ \\
\hline
\end{tabular}

$\varsigma_{4}=$ Common stand on climate change;

$\varsigma_{5}=$ Replacing thermal power fuel;

$\varsigma_{6}=$ Stand alone power projects;

$\varsigma_{7}=$ Use solar thermal;

$\varsigma_{8}=$ Perform energy audit.

The set of some basic causes is given by $\mathfrak{R}=\left\{\varrho_{1}, \varrho_{2}, \varrho_{3}, \varrho_{4}, \varrho_{5}, \varrho_{6}, \varrho_{7}\right\}$, where:

$\varrho_{1}=$ Over population;

$\varrho_{2}=$ Wastage of energy;

$\varrho_{3}=$ Poor infrastructure;

$\varrho_{4}=$ Poor dispersion scheme;

$\varrho_{5}=$ Major fortuities and instinctive disasters;

$\varrho_{6}=$ Wars and attacks;

$\varrho_{7}=$ Over consumption;

By applying the $\mathfrak{f n s}$-average operator on Table 1 the average values are given in Table 2 .

Comparison table for the above $\mathfrak{f n s}$-set is calculated by using Definition 4.1 given in Table 3.

The selection possibilities can be identified in the following order: $\varsigma_{3} \succ \varsigma_{5} \succ \varsigma_{1} \succ \varsigma_{2}=$ $\varsigma_{7} \succ \varsigma_{4}=\varsigma_{6} \succ \varsigma_{8}$. It can be easily seen from the above relation that the first three maximum resulting values are given by $\varsigma_{3}, \varsigma_{5}$ and $\varsigma_{1}$, which shows that we should work on the energy simulation and should buy energy efficient products with replacing thermal power fuel.

\section{Conclusion}

Fuzzy neutrosophic soft set theory has various applications in science and engineering, especially in the areas of neural networks, operations research, artificial intelligence and decision making. On this theme, we put forward the idea of $\mathfrak{f n} \mathfrak{n}$-mappings which is based on an $\mathfrak{f n s}$-element of an $\mathfrak{f n s}$-set in the $\mathfrak{f n s}$-topological space. We introduced the innovative 
idea of fixed points of $\mathfrak{f n s}$-mappings. We presented an outranking approach of an $\mathfrak{f n} \mathfrak{s}$-set in the decision making to reduce energy crises.

\author{
Conflict of interest \\ The authors declare that they have no conflict of interest.
}

\title{
Competing interests
}

The authors declare that they have no competing interest.

\section{Authors' contributions}

The authors contributed to each part of this paper equally. The authors read and approved the final manuscript.

\section{Publisher's Note}

Springer Nature remains neutral with regard to jurisdictional claims in published maps and institutional affiliations.

Received: 5 July 2017 Accepted: 11 December 2017 Published online: 19 February 2018

\section{References}

1. Zadeh, LA: Fuzzy sets. Inf. Control 8, 338-353 (1965)

2. Molodtsov, D: Soft set theory-first results. Comput. Math. Appl. 37, 19-31 (1999)

3. Maji, PK, Biswas, R, Roy, AR: Fuzzy soft sets. J. Fuzzy Math. 9(3), 589-602 (2001)

4. Çăgman, N, Çitak, F, Enginoglu, S: Fuzzy parameterized fuzzy soft set theory and its applications. Turk. J. Fuzzy Syst. 1(1), 21-35 (2010)

5. Zorlutuna, I, Atmaca, S: Fuzzy parametrized fuzzy soft topology. New Trends Math. Sci. 4(1), 142-152 (2016)

6. Atanassov, K: Intuitionistic fuzzy sets. Fuzzy Sets Syst. 20, 87-96 (1986)

7. Smarandache, F: Neutrosophy Neutrosophic Probability, Set and Logic. American Research Press, Rehoboth (1998)

8. Abbas, M, Khalid, A, Romaguera, S: Fixed points of fuzzy soft mappings. Appl. Math. Inf. Sci. 8(5), 2141-2147 (2014)

9. Abbas, M, Murtaza, G, Romaguera, S: On the fixed point theory of soft metric spaces. Fixed Point Theory Appl. 2016, $17(2016)$

10. Akram, M, Nawaz, S: Certain types of soft graphs. U.P.B. Sci. Bull., Ser. A 78(4), 67-82 (2016)

11. Akram, M, Nawaz, S: Fuzzy soft graphs with applications. J. Intell. Fuzzy Syst. 30(6), 3619-3632 (2016)

12. Arockiarani, I, Sumathi, IR, Martina, J: Jency, fuzzy neutrosophic soft topological spaces. Int. J. Math. Arch. 4(10), 225-238 (2013)

13. Feng, F, Akram, M, Davvaz, B, Fotea, VL: Attribute analysis of information systems based on elementary soft implications. Knowl.-Based Syst. 70, 281-292 (2014)

14. Feng, F, Jun, YB, Liu, X, Li, L: An adjustable approach to fuzzy soft set based decision making. J. Comput. Appl. Math. 234(1), 10-20 (2010)

15. Feng, F, Li, C, Davvaz, B, Ali, Ml: Soft sets combined with fuzzy sets and rough sets, a tentative approach. Soft Comput. 14(9), 899-911 (2010)

16. Feng, F, Liu, XY, Leoreanu-Fotea, $V$, Jun, YB: Soft sets and soft rough sets. Inf. Sci. 181(6), 1125-1137 (2011)

17. Wang, H, Smarandache, F, Zhang, YQ, Sunderraman, R: Single valued neutrosophic sets. Multispace and Multistructure 4, 410-413 (2010)

18. Karaaslan, F: Neutrosophic soft set with applications in decision making. Int. J. Inform. Sci. Intell. Syst. 4(2), 1-20 (2015)

19. Maji, PK: Neutrosophic soft set. Ann. Fuzzy Math. Inform. 5(1), 157-168 (2013)

20. Riaz, M, Naeem, K, Ahmad, MO: Novel concepts of soft sets with applications. Ann. Fuzzy Math. Inform. 13(2), 239-251 (2017)

21. Riaz, M, Naeem, K: Measurable soft mappings. Punjab Univ. J. Math. 48(2), 19-34 (2016)

22. Riaz, M, Fatima, Z: Certain properties of soft metric spaces. J. Fuzzy Math. 25(3), 543-560 (2017)

23. Riaz, M, Hashmi, MR: Fuzzy parameterized fuzzy soft topology with applications. Ann. Fuzzy Math. Inform. 13(5), 593-613 (2017)

24. Riaz, M, Hashmi, MR: Certain applications of fuzzy parameterized fuzzy soft sets in decision-making problems. Int. J. Algebra Statist. 5(2), 135-146 (2016)

25. Riaz, M, Hashmi, MR, Farooq, A: Fuzzy Parameterized Fuzzy Soft Metric spaces. Journal of Mathematical Analysis (2018, in press)

26. Samet, B: Coupled fixed point theorems for a generalized Meir-Keeler contraction in partially ordered metric spaces. Nonlinear Anal. 72, 4508-4517 (2010)

27. Jleli, M, Samet, B: Remarks on G-metric spaces and fixed point theorems. Fixed Point Theory Appl. 2012, 210 (2012)

28. Chen, CM, Lin, IJ: Fixed point theory of the soft Meir-Keeler type contractive mappings on a complete soft metric space. Fixed Point Theory Appl. 2015, 184 (2015)

29. Wardowski, D: On a soft mapping and its fixed points. Fixed Point Theory Appl. 2013, 182 (2013)

30. Al-Sharif, S, Alahmari, A, Al-Khaleel, M, Salem, A: New results on fixed points for an infinite sequence of mappings in g-metric space. Ital. J. Pure Appl. Math. 37, 517-540 (2017)

31. Ionescu, C, Rezapour, S, Samei, ME: Fixed points of some new contractions on intuitionistic fuzzy metric spaces. Fixed Point Theory Appl. 2013, 168 (2013)

32. Maji, PK, Biswas, R, Roy, AR: An application of soft sets in decision making problem. Comput. Math. Appl. 44(8-9), 1077-1083 (2002) 